\title{
Unilateral muscle contractions enhance creative thinking
}

\author{
Abraham Goldstein, Ketty Revivo, Michal Kreitler, and Nili Metuki \\ Bar-Ilan University, Ramat Gan, Israel
}

\begin{abstract}
Following the notion of relative importance of the right hemisphere $(\mathrm{RH})$ in creative thinking, we explored the possibility of enhancing creative problem solving by artificially activating the RH ahead of time using unilateral hand contractions. Participants attempted to complete the Remote Associates Test after squeezing a ball with either their left or right hand. As predicted, participants who contracted their left hand (thus activating the $\mathrm{RH})$ achieved higher scores than those who used their right hand and those who did not contract either hand. Our findings indicate that tilting the hemispheric balance toward the processing mode of one hemisphere by motor activation can greatly influence the outcome of thought processes. Regardless of the specific mechanism involved, this technique has the potential for acting as a therapeutic or remedial manipulation and could have wide applications in aiding individuals with language impairments or other disorders that are believed to be related to hemispheric imbalances.
\end{abstract}

Creative thinking entails the formation of associative links between otherwise unrelated concepts in order to solve problems in novel ways. As such, it is thought to require divergent thinking, which involves simultaneously considering various ideas, meanings, and approaches in order to derive a solution (Mednick, 1962). One possible approach to investigating the cognitive processes underlying the complex phenomenon of creative thinking is to examine the neural circuits involved. A widely held notion is that the information-processing style used by the right hemisphere (RH) facilitates this type of thinking (Fiore \& Schooler, 1998) and has been supported in a recent metaanalytic review (Mihov, Denzler, \& Förster, 2010). For example, the RH was found to be involved in reasoning about incompletely specified situations and in the maintenance of ambiguous mental representations (Goel et al., 2007), as well as in set-shift problems (Goel \& Vartanian, 2005). Analogously, psycholinguistic studies have shown that the RH induces diffuse activation of a broader semantic field that includes distant, unusual, and seemingly unrelated features, relative to the more constrained activation caused by the left hemisphere (LH; Jung-Beeman, 2005). For instance, studies have documented the relative importance of the RH in divergent semantic processing (Howard-Jones, Blakemore, Samuel, Summers, \& Claxton, 2005), in the generation of unusual verbs for presented nouns (Seger, Desmond, Glover, \& Gabrieli, 2000), and in comprehending novel metaphors (Arzouan, Goldstein, \& Faust, 2007).

Obviously, this approach does not maintain that creativity resides in the RH. As with any other high-level cognitive task, creative thinking involves a wide number of brain regions in both hemispheres working in a complex manner. Nevertheless, the wealth of findings associating RH activity with creative thinking indicate that the mode of operation of the RH (e.g., broad semantic fields, divergence) is particularly advantageous during such tasks; thus, conditions that enhance RH activity might lead to better performance.

The involvement of the RH in creativity has been demonstrated mainly in correlative studies showing a connection between RH activation and creative thinking performance. Our aim in this study was to explore the possibility of enhancing creative thinking by artificially activating or priming the $\mathrm{RH}$ ahead of time. Showing that a direct manipulation of the hemispheric balance has an effect on creativity would strengthen the case for the involvement of RH mechanisms. One method to achieve asymmetrical hemispheric activation is by way of unilateral muscle contractions. A number of studies have shown that contraction of the hand or face muscles has an effect on emotion and motivation, presumably by activating the motor and sensory areas of the hemisphere contralateral to the hand (Baumann, Kuhl, \& Kazén, 2005; Harmon-Jones, 2006; Peterson \& Harmon-Jones, 2008; Schiff, Esses, \& Lamon, 1992; Schiff \& Lamon, 1989, 1994; Shrira \& Martin, 2005). For example, consistent with the approach/ withdrawal motivational account of frontal asymmetry (Davidson, 1992), right-hand contractions, when compared with left-hand contractions, had the following effects: (1) less-negative emotional tones in descriptions of Thematic Apperception Test pictures (Schiff \& Lamon, 1994); (2) greater persistence in attempting to solve insoluble problems (Schiff, Guirguis, Kenwood, \& Her-

A. Goldstein, goldsa@mail.biu.ac.il 
man, 1998); (3) greater self-reported approach affect to a mildly positive radio editorial (Harmon-Jones, 2006); and (4) greater aggression toward insults (Peterson, Shackman, \& Harmon-Jones, 2008).

These behavioral and emotional consequences of unilateral contractions have been interpreted as resulting from the spreading of activation from sensorimotor areas to anterior regions of the activated hemisphere, affecting the asymmetry in those regions as well. In line with this interpretation, contractions of the right hand have been shown to induce less alpha synchronization in left frontal areas (Harmon-Jones, 2006), causing a relative LH/ RH asymmetry. Another possibility is that unilateral contractions induce lateral orientation of attention, which in turn leads to relatively greater activation in the contralateral hemisphere (Drake, 1991; Kinsbourne, 1970). In any case, unilateral hand contractions have been regarded as a manipulation of the relative balance of hemispheric activation.

Here, we tested whether unilateral hand contractions would have cognitive consequences for divergent thinking. In order to test the effects of unilateral hand contractions on creative thinking, we used a simple paper-and-pencil version of the Remote Associates Test (RAT; Mednick, 1968). In this widely used test of creative thinking, participants are presented with three words (e.g., tooth, potato, heart) and are asked to produce a solution word (e.g., sweet) that is related to all three words. Findings suggest that the RH is better able to retrieve and maintain semantic activation of unusual interpretations of the problem elements (Beeman \& Bowden, 2000); brain imaging and EEG data also agree with the notion of the relative importance of the RH in the RAT (Jung-Beeman et al., 2004). Unilateral hand contractions were operationalized by following the ball-squeezing procedure of Schiff et al. (1998). The hypothesis was that left-hand contractions would cause RH activation, thus shifting the hemispheric asymmetry toward the RH and leading to better scores in the creative thinking task.

\section{METHOD}

\section{Participants}

The 40 participants ( 24 female) were all right-handed volunteers who were native Hebrew speakers and were 21-35 years of age. The participants were randomly assigned to the control $(n=10)$, left-hand $(n=15)$, or right-hand $(n=15)$ squeeze condition. In order to rule out the effects of emotion, two additional groups (both $n \mathrm{~s}=10,5$ females in each group) contracted the right or left hand and subsequently completed a mood questionnaire without taking the creative thinking task.

\section{Procedure}

The participants were tested individually in a quiet room and were told that the research concerned the relationship between motor and cognitive activity. Participants in the contraction conditions squeezed a 7-cm-diameter rubber ball in their left or right hand. They were instructed to squeeze the ball as hard as they could while the other hand lay flat on the table or on their leg, with the palm facing down. Participants squeezed the ball for $45 \mathrm{sec}$ and relaxed for $15 \mathrm{sec}$. This was repeated four times. In the control condition, participants rested both palms on the table or leg and waited $4 \mathrm{~min}$ in a relaxed state before starting the experiment.
Immediately after this, participants performed two line-bisection trials as a manipulation check. On each trial, they were asked to mark the exact center of a $180-\mathrm{mm}$ black line printed horizontally on a white sheet of paper. The line was printed at midheight of the page and was closer to the right border on one trial and closer to the left border on the other. Participants used a fine-point pen to bisect the line as accurately as they could. Scores reflected the percent of deviation from the center of the line: Positive scores reflected a bias to the right side (stronger LH activation), and negative scores reflected a bias to the left side (stronger $\mathrm{RH}$ activation).

Following the line-bisection task, participants received a Hebrew pencil-and-paper version of the RAT (Nevo \& Levin, 1978), which was based on Mednick (1968). The test comprised 25 items ordered by difficulty. Each item contained three cue words, all associated with one word, such that they formed a compound word or a construct-state expression when attached to the target word (e.g., cue: pie, crab, pine; target: apple). Participants were instructed to solve as many problems as they could in $15 \mathrm{~min}$. They could skip problems or return to them at will. After this time, two additional line-bisection trials were performed. Last, in order to monitor possible motivational and emotional effects, participants' emotional state was assessed by means of the PANAS test (Watson, Clark, \& Tellegen, 1988), in which they indicated the extent to which they were experiencing 1 of a list of 20 emotions (10 positive, 10 negative) on a 5-point scale. Participants in the additional control conditions completed the PANAS test immediately after the manipulation.

\section{RESULTS}

The line-bisection index was more positive for righthand contractions $(M=1.79, S E=0.58)$ than for lefthand contractions $(M=0.11, S E=0.58)[t(28)=2.05$, $p<.05$, Cohen's $d=0.74]$, indicating that the manipulation had the expected hemispheric effect-right-hand contractions' leading to stronger LH activation. The handcontraction effect remained in the posttest line-bisection trials $[t(28)=2.28, p<.05$, Cohen's $d=0.83]$. An ANOVA conducted with number of problems solved in the RAT as the dependent variable and condition (right, left, control) as the independent variable revealed a significant effect of condition $\left[F(2,37)=7.35, p<.005, \eta_{\mathrm{p}}^{2}=.28\right]$. RAT scores were highest for left-hand contractions, lower for no contractions, and lowest for right-hand contractions (Figure 1). Post hoc tests (Duncan, 1955) revealed that the left-hand contraction group differed significantly from both the right-hand ( $p<.01$, Cohen's $d=0.59)$ and control $(p<.05$, Cohen's $d=0.32)$ groups. Overall, RAT scores were negatively correlated with line bisection $(r=$ $-.51, p<.001)$, indicating that the greater the RH bias, the higher the scores on the creative thinking task. To test for emotional effects, the correlation between RAT and PANAS scores was analyzed. RAT scores were inversely related to the negative-affect PANAS scores $(r=-.40$, $p<.05$ ) but were not correlated with the positive items of the PANAS. Participants who scored higher in the RAT test reported less negative emotion.

The possible impact of emotional aspects was further investigated by repeating the main ANOVA with the PANAS scores as covariates. The effect of hand contraction remained significant $[F(2,35)=5.91, p<.005]$, even when we controlled for positive and negative affect scores. In addition, ANOVAs of PANAS scores (positive and negative) of the right-hand and left-hand contraction condi- 


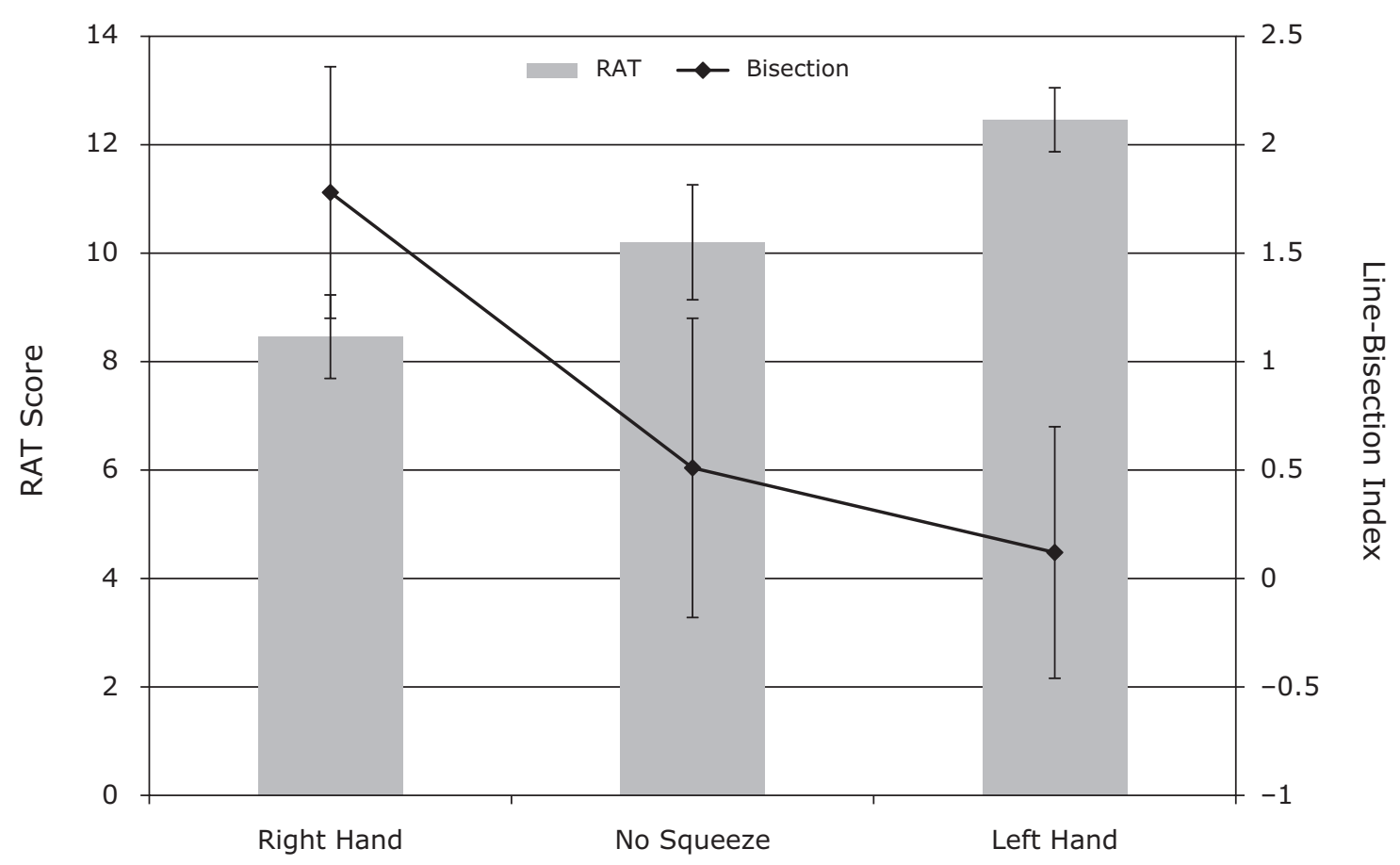

Figure 1. Remote Associates Test (RAT) scores (bars) and line-bisection indexes (lines) for right-hand, left-hand, and no-squeeze conditions. Error bars indicate the standard errors.

tions, after the creativity test and without it (Table 1), revealed no significant effects or interactions (all $p \mathrm{~s}>.2$ ).

\section{DISCUSSION}

Muscle activity in the hand is caused by activation of motor and sensory areas of the contralateral hemisphere. Previous studies have shown that the spreading of activation from these areas to other regions of the brain can have behavioral and emotional manifestations (Harmon-Jones, 2006; Schiff \& Lamon, 1994). Our results indicate that such effects are not limited to emotional responses but can have significant consequences in cognitive processing. Consistent with the findings regarding $\mathrm{RH}$ contribution to creative thinking - which are, for the most part, only correlative-we found that activating the RH by contracting the left hand resulted in better performance on a creative thinking task. Participants who squeezed the ball with their left hand achieved higher scores than those who used their right hand or who did not squeeze a ball at all.

Table 1

Mean Positive Affect-Negative Affect (PANAS) Scores Before and After the Remote Associates Test (RAT) in All Experimental Conditions

\begin{tabular}{llccccc}
\hline & & \multicolumn{2}{c}{ After RAT } & & \multicolumn{2}{c}{ Without RAT } \\
\cline { 3 - 4 } \cline { 6 - 7 } & & $M$ & $S D$ & & $M$ & $S D$ \\
\hline \multirow{2}{*}{ Negative } & Right hand & 19.6 & 9.1 & & 14.4 & 5.1 \\
& Left hand & 14.8 & 5.7 & & 15.8 & 5.3 \\
\multirow{2}{*}{ Positive } & Right hand & 24.1 & 5.8 & & 23.0 & 7.8 \\
& Left hand & 22.9 & 6.9 & & 23.9 & 6.8 \\
\hline
\end{tabular}

It might be argued that the results on the creative thinking task were due to changes in motivation and not in cognitive/semantic mode. Previous studies have shown how cuing a motivational state by contracting the extensor muscles of the arm or by cognitive nonsomatic cues can improve performance on creativity tests (Friedman \& Förster, 2002, 2005). However, in our case, the hemispheric effects associated with approach/withdrawal motivation predicted effects opposite to those we found. For example, Schiff et al. (1998) reported higher persistence in solving insoluble puzzles after right-hand muscle contractions, supposedly due to more approach motivation and positive affect. If such motivational biases occurred in our experiment, they did not lead to observable performance outcomes. In fact, our results indicate that RAT scores were lower after right-hand muscle contractions and were associated with negative affect. Thus, it appears that, in our study, the negative mood was secondary and might have been the result of self-evaluation of performance and not of hemispheric asymmetry. This is further corroborated by the lack of difference in PANAS scores between left- and right-contraction groups who did not participate in the problem-solving task.

The impact of unilateral muscle contraction on RAT scores seems to consist of both an enhancing effect of lefthand contraction and a detrimental effect of right-hand contraction. Creative thinking was significantly improved after squeezing with the left hand, relative to that in the no-squeeze condition. Thus, we suggest that increasing RH activity tilted the hemispheric balance toward divergent thinking and coarse coding, which are advantageous for solving these types of problems. Squeezing with the 
right hand resulted in poorer performance (although not statistically significant) than in the control condition, presumably by biasing toward fine coding and a convergent thinking style. Follow-up research should seek a double dissociation and test the effects of unilateral contraction on tasks demanding LH processing modes.

The detrimental effects of right-hand contractions, together with the correlation between RAT score and linebisection index (regardless of group), suggest that relative right/left activation, and not absolute hemispheric activation, is the key factor. Studies using unilateral vibrotactile stimulation (Bassel \& Schiff, 2001) have shown effects similar to those of unilateral contractions. Thus, the exact manipulation - motor or sensory - seems not to be crucial, as long as it produces a sufficient change in the hemispheric balance. Simple motor responses or short movement sequences that activate motor areas are not likely to tilt the hemispheric balance and spread to other areas and thus do not have motivational or cognitive consequences, as do those reported here. Also, it is not likely that the effects of hand contraction are related to hand dominance, since, in previous studies, they appeared to be the same when facial muscle contraction and forced nostril breathing were used (Schiff et al., 1998), whose dominance is not consistent with hand dominance.

The exact mechanism underlying the effects of unilateral muscle contraction on cognition and motivation is unknown. One possible mechanism could be the spreading of alpha-rhythm desynchronization. Voluntary movement and somatosensory stimulation are known to reduce the power of mu rhythms (which are in the same frequency range as alpha: $8-13 \mathrm{~Hz}$ ) over sensorimotor cortex (Pfurtscheller, Neuper, Andrew, \& Edlinger, 1997). Contraction of the right hand has been shown to induce less alpha synchronization in left sites that spreads from central areas to frontal areas (Harmon-Jones, 2006), causing a relative LH/RH asymmetry. The LH bias resulted in approach motivational behavior - for example, anger toward insult (Peterson et al., 2008) — consistent with the approach/ withdrawal motivation model of frontal asymmetry (Davidson, 1992). The cognitive effects reported in the present study might be explained in a similar fashion. If lefthand squeezing led to an alpha-range desynchronization in RH sensorimotor areas, this activity pattern is likely to have spread to other anterior and posterior regions in that hemisphere, not only to the frontal areas involved in motivation.

Recently, hemispheric asymmetry in resting-state EEG has been shown to be related to individual differences in insight-problem solving (Kounios et al., 2008). Participants who were more likely to use insight during anagram problem solving had lower alpha-EEG power at right dorsal-frontal areas and greater alpha power at left inferior-frontal and left anterior-temporal sites. Other studies have found hemispheric differences in alpha-band transient event-related synchronization and desynchronization during creative thinking tasks (Grabner, Fink, \& Neubauer, 2007; Jung-Beeman et al., 2004; Sandkühler \& Bhattacharya, 2008). Whether unilateral muscle con- tractions influence creative problem solving by spreading alpha activity from motor to cognitive areas should be tested using electrophysiological measures. This would also assist in defining the time course and duration of the effect.

Another possible mechanism underlying our findings could be an indirect effect of spatial attention. Squeezing with one hand may shift the attention to that side of the body and activate the contralateral hemisphere responsible for that part of space/body. This is reflected in the results reported here on the line-bisection task. Inducing attention orientation to one side might lead to activation of the contralateral hemisphere (Drake, 1991; Kinsbourne, 1970). A recent study (Bultitude \& Woods, 2010) has shown that adaptation to leftward-shifting prisms, which has a rightward visuomotor aftereffect, can influence the global-local nonspatial attentional bias. Thus, it is possible that unilateral hand contractions have a similar aftereffect, resulting in a bias toward the attentional style of the contralateral hemisphere.

The general right-left dichotomy is, of course, simplistic and should be used cautiously. Unquestionably, creativity does not reside or originate in the $\mathrm{RH}-\mathrm{or}$, for that matter, in any particular brain area. Both hemispheres are involved in every mental task we perform, working in concert, each providing particular information relevant to its processing style. Nevertheless, our findings indicate that tilting the hemispheric balance toward the processing mode of one hemisphere artificially by motor activation can greatly influence the outcome of the thought process. Regardless of the specific mechanism involved, if our results are replicated in other tasks and cognitive domains, this technique has the potential to act as a therapeutic or remedial manipulation and could have wide applications - for example, in aiding individuals with language impairments or other disorders believed to be related to hemispheric imbalances.

\section{AUTHOR NOTE}

Correspondence concerning this article should be addressed to A. Goldstein, Gonda Brain Research Center, Bar-Ilan University, Ramat Gan, 52900, Israel (e-mail: goldsa@mail.biu.ac.il).

$$
\text { Note-Accepted by Cathleen M. Moore's editorial team. }
$$

\section{REFERENCES}

Arzouan, Y., Goldstein, A., \& Faust, M. (2007). Dynamics of hemispheric activity during metaphor comprehension: Electrophysiological measures. NeuroImage, 36, 222-231. doi:10.1016/j.neuroimage 2007.02.015

BASSEL, C., \& SCHIFF, B. B. (2001). Unilateral vibrotactile stimulation induces emotional biases in cognition and performance. Neuropsychologia, 39, 282-287. doi:10.1016/S0028-3932(00)00116-0

Baumann, N., KuHL, J., \& KAZÉN, M. (2005). Left-hemispheric activation and self-infiltration: Testing a neuropsychological model of internalization. Motivation \& Emotion, 29, 135-163. doi:10.1007/ s11031-005-9439-X

Beeman, M. J., \& Bowden, E. M. (2000). The right hemisphere maintains solution-related activation for yet-to-be-solved problems. Memory \& Cognition, 28, 1231-1241.

Bultitude, J. H., \& Woods, J. M. (2010). Adaptation to leftward-shifting prisms reduces the global processing bias of healthy individuals. 
Neuropsychologia, 48, 1750-1756. doi:10.1016/j.neuropsychologia .2010 .02 .024

DAVIDSON, R. J. (1992). Anterior cerebral asymmetry and the nature of emotion. Brain \& Cognition, 20, 125-151. doi:10.1016/0278 -2626(92)90065-T

DRAKe, R. A. (1991). Processing persuasive arguments: Recall and recognition as a function of agreement and manipulated activation asymmetry. Brain \& Cognition, 15, 83-94. doi:10.1016/0278 -2626(91)90017-3

DunCAN, D. B. (1955). Multiple range and multiple $F$ tests. Biometrics, 11, 1-42.

Fiore, S. M., \& Schooler, J. W. (1998). Right hemisphere contributions to creative problem solving: Converging evidence for divergent thinking. In M. Beeman \& C. Chiarello (Eds.), Right hemisphere language comprehension: Perspectives from cognitive neuroscience (pp. 349-371). Mahwah, NJ: Erlbaum.

Friedman, R. S., \& Förster, J. (2002). The influence of approach and avoidance motor actions on creative cognition. Journal of Experimental Social Psychology, 38, 41-55. doi:10.1006/jesp.2001.1488

Friedman, R. S., \& Förster, J. (2005). Effects of motivational cues on perceptual asymmetry: Implications for creativity and analytical problem solving. Journal of Personality \& Social Psychology, 88, 263-275. doi:10.1037/0022-3514.88.2.263

Goel, V., Tierney, M., Sheesley, L., Bartolo, A., Vartanian, O., \& Grafman, J. (2007). Hemispheric specialization in human prefrontal cortex for resolving certain and uncertain inferences. Cerebral Cortex, 17, 2245-2250. doi:10.1093/cercor/bhl132

Goel, V., \& Vartanian, O. (2005). Dissociating the roles of right ventral lateral and dorsal lateral prefrontal cortex in generation and maintenance of hypotheses in set-shift problems. Cerebral Cortex, 15, 1170-1177. doi:10.1093/cercor/bhh217

Grabner, R. H., Fink, A., \& Neubauer, A. C. (2007). Brain correlates of self-rated originality of ideas: Evidence from event-related power and phase-locking changes in the EEG. Behavioral Neuroscience, 121, 224-230. doi:10.1037/0735-7044.121.1.224

HARMON-JONES, E. (2006). Unilateral right-hand contractions cause contralateral alpha power suppression and approach motivational affective experience. Psychophysiology, 43, 598-603. doi:10.1111/j.1469 -8986.2006.00465.x

Howard-Jones, P. A., Blakemore, S.-J., Samuel, E. A., Summers, I. R., \& Claxton, G. (2005). Semantic divergence and creative story generation: An fMRI investigation. Cognitive Brain Research, 25, 240-250. doi:10.1016/j.cogbrainres.2005.05.013

Jung-Beeman, M. (2005). Bilateral brain processes for comprehending natural language. Trends in Cognitive Sciences, 9, 712-718. doi:10.1016/j.tics.2005.09.009

Jung-Beeman, M., Bowden, E. M., Haberman, J., Frymiare, J. L., Arambel-Liu, S., Greenblatt, R., ET AL. (2004). Neural activity when people solve verbal problems with insight. PLoS Biology, 2, 500-510. doi:10.1371/journal.pbio.0020097

Kinsbourne, M. (1970). The cerebral basis of lateral asymmetries in attention. Acta Psychologica, 33, 193-201. doi:10.1016/0001 $-6918(70) 90132-0$
Kounios, J., Fleck, J. I., Green, D. L., Payne, L., Stevenson, J. L., Bowden, E. M., \& Jung-Beeman, M. (2008). The origins of insight in resting-state brain activity. Neuropsychologia, 46, 281-291. doi:10.1016/j.neuropsychologia.2007.07.013

Mednick, S. A. (1962). The associative basis of the creative process. Psychological Review, 69, 220-232. doi:10.1037/h0048850

Mednick, S. A. (1968). Remote Associates Test. Journal of Creative Behavior, 2, 213-214.

Minov, K. M., Denzler, M., \& Förster, J. (2010). Hemispheric specialization and creative thinking: A meta-analytic review of lateralization of creativity. Brain \& Cognition, 72, 442-448. doi:10.1016/ j.bandc.2009.12.007

Nevo, B., \& Levin, I. (1978). Remote Associates Test: Assessment of creativity in Hebrew. Megamot, 24, 87-98.

Peterson, C. K., \& Harmon-Jones, E. (2008). Proneness to hypomania predicts EEG coherence between left motor cortex and left prefrontal cortex. Biological Psychology, 78, 216-219. doi:10.1016/ j.biopsycho.2008.01.011

Peterson, C. K., Shackman, A. J., \& Harmon-Jones, E. (2008). The role of asymmetrical frontal cortical activity in aggression. Psychophysiology, 45, 86-92. doi:10.1111/j.1469-8986.2007.00597.x

Pfurtscheller, G., Neuper, C., Andrew, C., \& Edlinger, G. (1997). Foot and hand area mu rhythms. International Journal of Psychophysiology, 26, 121-135. doi:10.1016/S0167-8760(97)00760-5

SANDKÜHLER, S., \& Bhattacharya, J. (2008). Deconstructing insight: EEG correlates of insightful problem solving. PLOS ONE, 3, e1459. doi:10.1371/journal.pone.0001459

Schiff, B. B., Esses, V. M., \& LAMON, M. (1992). Unilateral facial contractions produce mood effects on social cognitive judgments. Cognition \& Emotion, 6, 357-368. doi:10.1080/02699939208409691

Schiff, B. B., Guirguis, M., Kenwood, C., \& Herman, C. P. (1998). Asymmetrical hemispheric activation and behavioral persistence: Effects of unilateral muscle contractions. Neuropsychology, 12, 526532.

Schiff, B. B., \& LAMON, M. (1989). Inducing emotion by unilateral contraction of facial muscles: A new look at hemispheric specialization and the experience of emotion. Neuropsychologia, 27, 923-935. doi:10.1016/0028-3932(89)90068-7

SCHIFF, B. B., \& LAMON, M. (1994). Inducing emotion by unilateral contraction of hand muscles. Cortex, 30, 247-254.

Seger, C. A., Desmond, J. E., Glover, G. H., \& Gabrieli, J. D. E. (2000). Functional magnetic resonance imaging evidence for righthemisphere involvement in processing unusual semantic relationships. Neuropsychology, 14, 361-369.

Shrira, I., \& Martin, L. L. (2005). Stereotyping, self-affirmation, and the cerebral hemispheres. Personality \& Social Psychology Bulletin, 31, 846-856. doi: $10.1177 / 0146167204272232$

Watson, D., Clark, L. A., \& Tellegen, A. (1988). Development and validation of brief measures of positive and negative affect: The PANAS scales. Journal of Personality \& Social Psychology, 54, 1063-1070.

(Manuscript received January 13, 2010; revision accepted for publication July 15, 2010.) 